On comparing the second and fourth rows of Table 3 we see that for the first four characteristic values the results obtained from the modified Method II are better than those given by the unmodified one for $N=9$. Other computations have shown that this result does not hold for $m \neq 2$ if the class of admissible function is defined so as to have continuous derivatives at the points $x=x_{i}$ and if the matrix $D$ is evaluated as in Method II.

In the third row of Table 3 the matrix $D$ is evaluated as in Method II where the admissible functions are defined as for the first two rows.

The numerical results, although obtained only for a particular simple example, would seem to justify the use of the methods discussed above for the solution of more complicated Sturm-Liouville problems. They should be supplemented by a test for the accuracy of the results obtained for the characteristic values and characteristic functions applicable to problems for which the solution is not known. Various such tests are under investigation and will be reported on subsequently.

Digital Computer Laboratory

C. C. Farrington

University of Illinois

Urbana, Illinois

University of California

R. T. GREGORY

Goleta, California

Digital Computer Laboratory

A. H. TAUB

University of Illinois

Urbana, Illinois

This work was supported in part by the National Science Foundation under Grant G-1221.

1. F. B. Hildebrand, Introduction to Numerical Analysis, McGraw-Hill Book Co., New York, 1956, p. 241.

2. R. T. GREGORY, "A numerical solution to a class of Sturm-Liouville systems," University of Illinois thesis, 1955 .

3. R. T. GREGORY, "Computing eigenvalues and eigenvectors of a symmetric matrix on the ILLIAC," $M T A C$, v. 7, 1953, p. 215-220.

\title{
On the Numerical Evaluation of the Stokes' Stream Function
}

1. Introduction. In the study of axially symmetric problems in fluid dynamics, the Stokes' stream function, that is, the function which satisfies

$$
u_{x x}-\frac{1}{y} u_{y}+u_{y y}=0, \quad y \neq 0,
$$

is of considerable interest. This function is constant on the streamlines.

The problem to be considered is a Dirichlet type problem. Let $G$ be a closed, bounded, simply connected plane region whose interior is denoted by $R$ and whose boundary curve is denoted by $S$. Let $G$ not contain any point where $y=0$. 
Let $g(x, y)$ be defined and continuous on $S$. The problem then is to produce a function $u(x, y)$ such that
a) $u(x, y) \equiv g(x, y)$, on $S$, and
b) $u(x, y)$ satisfies $(1.1)$ in $R$.

Under general conditions, there exists a unique solution (Bernstein [1] and Courant and Hilbert [3]), and only such cases will be considered. However, the analytical determination of $u(x, y)$ is quite another story from that of its existence and usually offers what are at present insurmountable problems. The approach here, then, will be from a numerical analysis point of view.

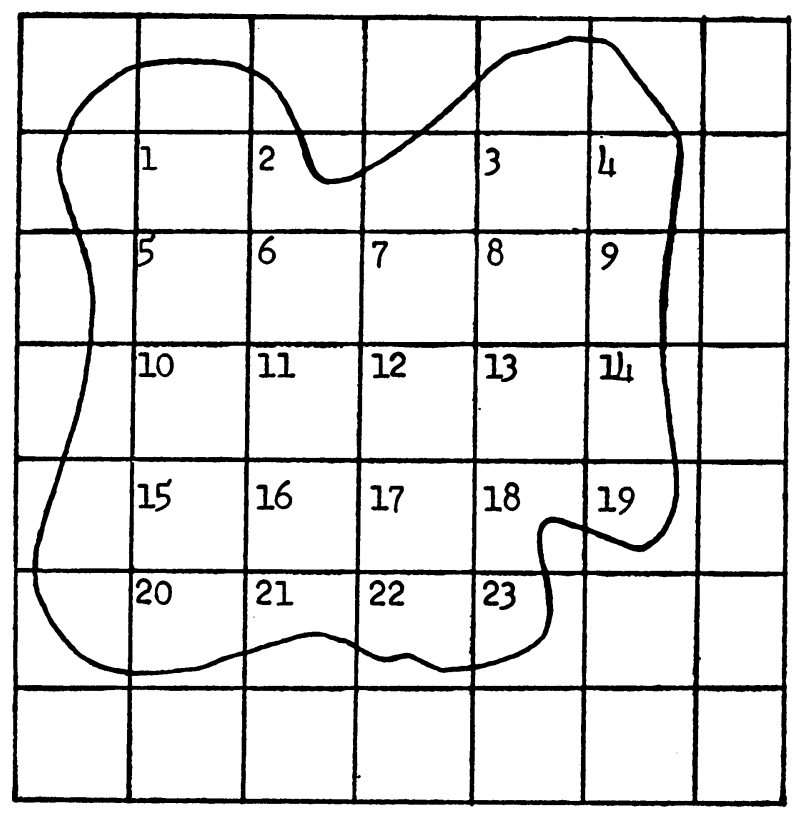

DIAGRAM 1

Let $h$ be a fixed positive constant called the mesh size. Let $\left(x_{0}, y_{0}\right)$ be an arbitrary, but fixed point of $G$ and denote by $G_{h}$ the set of all points of form $\left(x_{0}+m h, y_{0}+n h\right)$, contained in $G$, where $m$ and $n$ are integers. Two points $\left(x_{1}, y_{1}\right)$ and $\left(x_{2}, y_{2}\right)$ of $G$ are called adjacent if and only if $\left(x_{2}-x_{1}\right)^{2}+\left(y_{2}-y_{1}\right)^{2}=h^{2}$. $R_{h}$, the interior of $G_{h}$, is the set of all points of $G_{h}$ whose four adjacent points belong to $G_{h}$. $S_{h}$, the boundary of $G_{h}$, called the lattice boundary, is defined by $S_{h}=G_{h}-R_{h}$. It is also assumed that any pair of points of $R_{h}$ can be joined by a connected polygonal arc consisting of straight line segments which join adjacent points of $R_{h}$.

The technique proposed will involve the application of a difference equation to yield a system of linear equations whose solution is an approximation to the solution of the formulated Dirichlet problem at the points of $G_{h}$.

The question of solving the linear system will not be considered. Systems with unique solutions may be approached by means of Cramer's rule, Gauss' Elimina- 
tion Procedure, matrix inversion, relaxation, iteration, gradient methods, and other techniques (Geiringer [4], Greenspan [6], Milne [7], Shortley et al [8], NBS [9], Young $[13,14])$.

The author is indebted to $D$. M. Young for valuable criticisms and suggestions.

2. General Method. Suppose $G_{h}$ consists of $n$ points. Number these points in a one-to-one fashion with the integers $1,2,3, \cdots, n$. Denote the coordinates of the point numbered $k$ by $\left(x_{k}, y_{k}\right)$ and the unknown stream function at $\left(x_{k}, y_{k}\right)$ by $u\left(x_{k}, y_{k}\right) \equiv u_{k}$, for $k=1,2, \cdots, n$.

Let $\left(x_{i}, y_{i}\right)$ be an arbitrary point of $S_{h}$, the lattice boundary. Approximate $u_{i}$ by $g\left(x^{\prime}, y^{\prime}\right)$, where $\left(x^{\prime}, y^{\prime}\right)$ is the nearest point of $S$ to $\left(x_{i}, y_{i}\right)$. If $\left(x^{\prime}, y^{\prime}\right)$ is not unique, choose any one of the set of nearest points and use it. The problem of

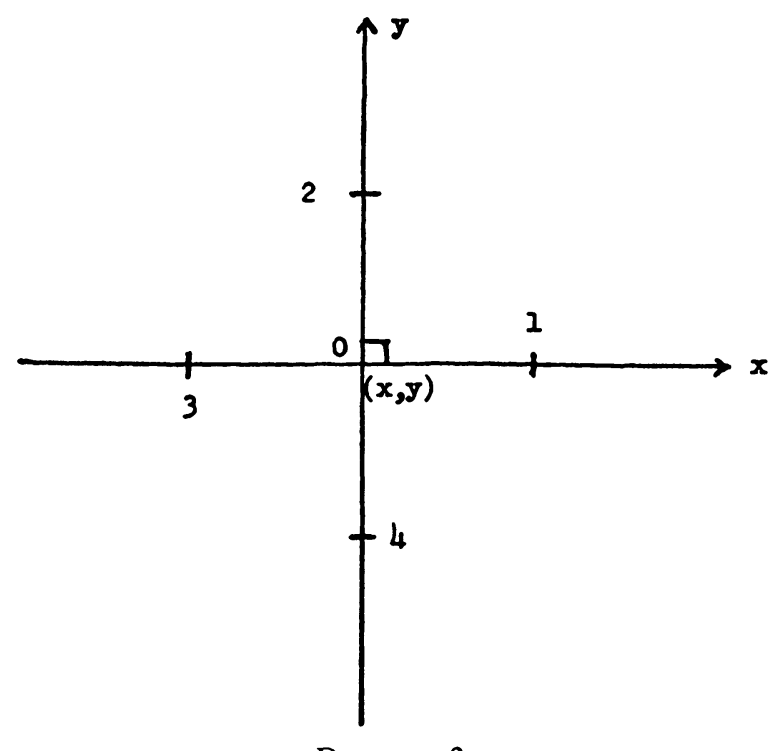

DIAGRAM 2

finding numerical approximations to $u(x, y)$ on the lattice boundary is, though crudely done, adequate for present purposes. For diagram 1 this means that at the points $1,2,3,4,5,7,9,10,14,15,19,20,21,22,23$ the values of the stream function have been approximated by values at nearby points of $S$.

We then require that at each point $\left(x_{i}, y_{i}\right)$ of $R_{h}$, the function $u$ satisfy

$$
\begin{aligned}
4 u\left(x_{i}, y_{i}\right)-u\left(x_{i}+h, y_{i}\right) & -\left(1-\frac{h}{2 y_{i}}\right) u\left(x_{i}, y_{i}+h\right) \\
& -u\left(x_{i}-h, y_{i}\right)-\left(1+\frac{h}{2 y_{i}}\right) u\left(x_{i}, y_{i}-h\right)=0 .
\end{aligned}
$$

It is convenient, in practice, to use the subscript notation. For example, for diagram 1 , if $i=12,(2.1)$ becomes

$$
4 u_{12}-u_{13}-\left(1-\frac{h}{2 y_{12}}\right) u_{7}-u_{11}-\left(1+\frac{h}{2 y_{12}}\right) u_{17}=0 .
$$


Note that $u_{12}, u_{13}, u_{11}, u_{17}$ are unknowns while $u_{7}$ is a constant determined by the method for points of $S_{h}$. Application of (2.1) to each point of $R_{h}$ thereby results in a system of linear equations which, when solved, yields the remaining numerical approximations.

Equation (2.1) is only one of a variety of possible difference equations which can be developed, as described in the next section.

3. Development of a General Difference Equation. We shall seek a solution $u(x, y)$ which is of class $C^{4}$ and use the notation $\partial^{i+j} u / \partial x^{i} \partial y^{j} \equiv u_{i, j}, i+j \leq 4$, and $A_{i j} \equiv u_{i, j} / i ! j !$.

Let $(x, y)$ be an arbitrary point of $R_{h}$. The aim will be to develop a "5-point formula," so consider here only $(x, y)$ and its four adjacent points. Let $(x, y)$, $(x+h, y),(x, y+h),(x-h, y)$, and $(x, y-h)$ be denoted, respectively, by $0,1,2,3,4$, as in diagram 2 . Hence $\left(x_{0}, y_{0}\right)=(x, y),\left(x_{1}, y_{1}\right)=(x+h, y)$, $\left(x_{2}, y_{2}\right)=(x, y+h),\left(x_{3}, y_{3}\right)=(x-h, y),\left(x_{4}, y_{4}\right)=(x, y-h)$. Also let $O\left(h^{n}\right)$ represent any and all functions of order at least $n$ in $h$.

In order to deduce a difference equation which approximates (1.1), let

$$
L(u)=\sum_{0}^{4} \alpha_{i} u_{i}
$$

By use of the finite Taylor series expansions about $\left(x_{0}, y_{0}\right)$ for the various $u_{i}$ and by the definition of $A_{i j}$, (3.1) becomes

$$
\begin{aligned}
L(u)=A_{00}\left(\alpha_{0}+\alpha_{1}+\alpha_{2}\right. & \left.+\alpha_{3}+\alpha_{4}\right)+h\left[A_{10}\left(\alpha_{1}-\alpha_{3}\right)+A_{01}\left(\alpha_{2}-\alpha_{4}\right)\right] \\
+ & h^{2}\left[A_{20}\left(\alpha_{1}+\alpha_{3}\right)+A_{02}\left(\alpha_{2}+\alpha_{4}\right)\right] \\
& +h^{3}\left[A_{30}\left(\alpha_{1}-\alpha_{3}\right)+A_{03}\left(\alpha_{2}-\alpha_{4}\right)\right]+O .\left(h^{4}\right)
\end{aligned}
$$

Equation (1.1) may be rewritten as $y u_{2,0}-u_{0,1}+y u_{0,2}=0$. Since $u$ is assumed to be of class $C^{4}$, further differentiation of (1.1) yields

$$
\begin{aligned}
& y u_{3,0}-u_{1,1}+y u_{1,2}=0 \\
& u_{2,0}+y u_{2,1}+y u_{0,3}=0
\end{aligned}
$$

Rewriting these in terms of the $A_{i j}$ 's and solving, one finds

$$
A_{20}=\frac{A_{01}}{2 y}-A_{02} ; \quad A_{30}=\frac{A_{11}}{6 y}-\frac{A_{12}}{3} ; \quad A_{21}=-3 A_{03}+\frac{A_{02}}{y}-\frac{A_{01}}{2 y^{2}}
$$

Substitution of (3.3) into (3.2) yields

$$
\begin{aligned}
L(u)= & A_{00}\left(\alpha_{0}+\alpha_{1}+\alpha_{2}+\alpha_{3}+\alpha_{4}\right) \\
& +h\left[A_{01}\left(\frac{\alpha_{1} h}{2 y}+\frac{\alpha_{3} h}{2 y}+\alpha_{2}-\alpha_{4}\right)+A_{10}\left(\alpha_{1}-\alpha_{3}\right)\right] \\
& +h^{2}\left[A_{02}\left(\alpha_{2}+\alpha_{4}-\alpha_{1}-\alpha_{3}\right)\right] \\
& \quad+h^{3}\left[A_{30}\left(\alpha_{1}-\alpha_{3}\right)+A_{03}\left(\alpha_{2}-\alpha_{4}\right)\right]+O\left(h^{4}\right) .
\end{aligned}
$$


Let

where

$$
\begin{aligned}
\alpha_{0}+\alpha_{1}+\alpha_{2}+\alpha_{3}+\alpha_{4} & =\epsilon_{1} \\
\frac{\alpha_{1} h}{2 y}+\frac{\alpha_{3} h}{2 y}+\alpha_{2}-\alpha_{4} & =\epsilon_{2} \\
\alpha_{1}-\alpha_{3} & =\epsilon_{3} \\
\alpha_{2}+\alpha_{4}-\alpha_{1}-\alpha_{3} & =\epsilon_{4} \\
\alpha_{2}-\alpha_{4} & =\epsilon_{5}
\end{aligned}
$$

$$
\epsilon_{1}=O\left(h^{4}\right), \quad \epsilon_{2}=O\left(h^{3}\right), \quad \epsilon_{3}=O\left(h^{3}\right), \quad \epsilon_{4}=O\left(h^{2}\right), \quad \epsilon_{5}=O(h) .
$$

The solution to system (3.5) is

$$
\begin{gathered}
\alpha_{1}=\frac{\epsilon_{2} y}{h}-\frac{\epsilon_{5} y}{h}+\frac{\epsilon_{3}}{2} \quad \alpha_{2}=\frac{\epsilon_{2} y}{h}+\frac{\epsilon_{4}}{2}+\frac{\epsilon_{5}}{2}-\frac{\epsilon_{5} y}{h} \\
\alpha_{3}=\frac{\epsilon_{2} y}{h}-\frac{\epsilon_{5} y}{h}-\frac{\epsilon_{3}}{2} \quad \alpha_{4}=\frac{\epsilon_{2} y}{h}+\frac{\epsilon_{4}}{2}-\frac{\epsilon_{5}}{2}-\frac{\epsilon_{5} y}{h} \\
\alpha_{0}=\epsilon_{1}-\epsilon_{4}+4 \frac{\epsilon_{5} y}{h}-4 \frac{\epsilon_{2} y}{h} .
\end{gathered}
$$

Finally, setting (3.1) equal to zero and substituting (3.6) in (3.1) yield the general difference equation (3.7) which approximates the differential equation (1.1)

$$
\begin{aligned}
\left(\epsilon_{1}-\epsilon_{4}\right. & \left.+\frac{4 \epsilon_{5} y}{h}-\frac{4 \epsilon_{2} y}{h}\right) u_{0}+\left(\frac{\epsilon_{2} y}{h}-\frac{\epsilon_{5} y}{h}+\frac{\epsilon_{3}}{2}\right) u_{1} \\
+\left(\frac{\epsilon_{2} y}{h}+\frac{\epsilon_{4}}{2}+\frac{\epsilon_{5}}{2}-\frac{\epsilon_{5} y}{h}\right) u_{2}+ & \left(\frac{\epsilon_{2} y}{h}-\frac{\epsilon_{5} y}{h}-\frac{\epsilon_{3}}{2}\right) u_{3} \\
& +\left(\frac{\epsilon_{2} y}{h}+\frac{\epsilon_{4}}{2}-\frac{\epsilon_{5}}{2}-\frac{\epsilon_{5} y}{h}\right) u_{4}=0 .
\end{aligned}
$$

The process, then, was to find $\alpha_{i}$ such that: $L(u)+O\left(h^{4}\right) \equiv 0$, where $u$ satisfies (1.1), and to then use $L(u)=0$ for the general approximation.

Equation (2.1), which will be used throughout, is deduced from (3.7) by letting $\epsilon_{1}=\epsilon_{2}=\epsilon_{3}=\epsilon_{4}=0, \epsilon_{5}=h$, by dividing through by $y$, and by noting $u_{0}=u(x, y)$, $u_{1}=u(x+h, y), u_{2}=u(x, y+h), u_{3}=u(x-h, y), u_{4}=u(x, y-h)$.

Other difference equations may be constructed by selecting the $\epsilon_{i}$ 's differently. One of these of particular interest is one which would yield a linear system which has a symmetric coefficient matrix. This may be done in the following manner. Number the points as in diagram 1 so that the numbers increase from left to right and also increase if one reads down any column of points. Apply difference equation

$$
\begin{aligned}
& u_{0}\left(+\frac{2}{y}+\frac{2}{2 y+h}+\frac{2}{2 y-h}\right)-\frac{u_{1}}{y}-\frac{u_{3}}{y} \\
& \quad-u_{2}\left(\frac{2}{2 y+h}\right)-u_{4}\left(\frac{2}{2 y-h}\right)=0, \quad|2 y| \neq h
\end{aligned}
$$


to the points of $R_{h}$. Start with the first, or highest, row of points; traverse the row from left to right; proceed to the next row; traverse the row from left to right; proceed to the next row, etc.; continue the process until all the points of $R_{h}$ have been used. Equation (3.8) may be constructed by a numerical procedure for certain self-adjoint elliptic differential equations (Young [15]), or directly from (3.7) by letting

$$
\begin{aligned}
& \epsilon_{1}=0, \quad \epsilon_{2}=\frac{-h}{y^{2}}+\frac{4 h}{(2 y+h)(2 y-h)}, \quad \epsilon_{3}=0, \\
& \epsilon_{4}=\frac{-8 y}{(2 y+h)(2 y-h)}+\frac{2}{y}, \quad \epsilon_{5}=\frac{+4 h}{(2 y+h)(2 y-h)} .
\end{aligned}
$$

4. A Theorem on an Error Bound. In this section, let $u(x, y)$ be the solution of the Dirichlet problem being considered and $U(x, y)$ the solution of the numerical method described in section 2 . This means that

$$
-4 U_{0}+U_{1}+U_{3}+\left(1-\frac{h}{2 y}\right) U_{2}+\left(1+\frac{h}{2 y}\right) U_{4}=0
$$

for any arrangement of points of $G_{h}$ like that of diagram 2 .

LEMMA 1. The solution of the system of linear equations which results by application of the process of section 2 with $y \neq 0$ and $\left|\frac{h}{y}\right|<1$ is unique.

Proof. It is sufficient to show that the determinant of the system of linear equations is not zero and this is done by demonstrating that the only solution of the homogeneous system which results by considering $g(x, y) \equiv 0$ on $S$ is the zero vector. Suppose then there exists a nontrivial solution for the homogeneous system. For some point of $R_{h}, U \neq 0$. Suppose $U>0$. Let the largest value $M$ occur at $\left(x_{0}, y_{0}\right)$. Then

$$
4 U_{0}=U_{1}+U_{3}+\left(1-\frac{h}{2 y}\right) U_{2}+\left(1+\frac{h}{2 y}\right) U_{4},
$$

and

$$
M=U_{0} \geq U_{i}, \quad i=1,2,3,4 .
$$

Case 1. $U_{2}=U_{4}$. Then (4.1) becomes

$$
4 U_{0}=U_{1}+U_{2}+U_{3}+U_{4}
$$

But (4.3) and (4.4) imply $U_{0}=U_{1}=U_{2}=U_{3}=U_{4}=M$. Hence one can apply the same argument at $\left(x_{1}, y_{1}\right)$ and continue in a finite number of steps to show that $U$ at a boundary point is equal to $M$. This is a contradiction since $g(x, y) \equiv 0$ on $S$ and the method described for points of $S_{h}$ implies $U$ at all lattice boundary points is zero. 
Case 2. $U_{2}>U_{4}$. Let $U_{4}=U_{2}-k, k>0$. Then

and

$$
4 U_{0}=U_{1}+2 U_{2}+U_{3}-k\left(1+\frac{h}{2 y}\right),
$$

$$
4 U_{0}<U_{1}+2 U_{2}+U_{3}, \text { since } 1+\frac{h}{2 y}>0 .
$$

But this is impossible since $U_{1} \leq U_{0}, U_{2} \leq U_{0}, U_{3} \leq U_{0}$.

Case 3. $U_{4}>U_{2}$. The discussion is analogous to that for Case 2 and leads to a contradiction.

Hence, in all three cases, the assumption that $U>0$ yields a contradiction. Contradictions are similarly reached if $U<0$ is assumed. Hence the only solution is the trivial solution. This completes the proof.

Using the same ideas and techniques first set forth by Gershgorin [5], we apply these by assuming in all that follows that $\sigma=h / 2 y,|\sigma|<1$ and by defining

$$
\begin{aligned}
\lambda[u(x, y)] \equiv h^{-2}\{- & 4 u(x, y)+u(x+h, y)+u(x-h, y) \\
& \left.+\left(1-\frac{h}{2 y}\right) u(x, y+h)+\left(1+\frac{h}{2 y}\right) u(x, y-h)\right\} .
\end{aligned}
$$

Lemma 2. If $\lambda[v] \leq 0$ on $R_{h}$ and $v \geq 0$ on $S_{h}$, then $v \geq 0$ on $R_{h}$.

Proof. Suppose $v<0$ for some point of $R_{h}$. Then there exists a point $\left(x_{0}, y_{0}\right)$ of $R_{h}$ where $v$ is a minimum. Hence

$$
v\left(x_{0}, y_{0}\right)<0 \text {, and, } v\left(x_{0}, y_{0}\right) \leq v(x, y) \text {, for all }(x, y) \text { of } R_{h} .
$$

Now, $\lambda[v] \leq 0$, by assumption, so that, equivalently

$$
v_{0} \geq \frac{1}{4}\left[v_{1}+\left(1-\frac{h}{2 y}\right) v_{2}+v_{3}+\left(1+\frac{h}{2 y}\right) v_{4}\right] \text {. }
$$

But (4.6) and (4.7) imply, in a manner similar to that used in Lemma 1, that $v_{0}=v_{1}=v_{2}=v_{3}=v_{4}$. In a finite number of steps this leads to the result that $v<0$ for some point of $S_{h}$, which is a contradiction. This completes the proof.

Lemma 3. If $-\left|\lambda\left[v_{1}\right]\right| \geq \lambda\left[v_{2}\right]$ in $R_{h}$ and $\left|v_{1}\right| \leq v_{2}$ on $S_{h}$, then $\left|v_{1}\right| \leq v_{2}$ on $R_{h}$.

Proof. $v_{2}-v_{1} \geq 0$ on $S_{h}$ and $\lambda\left[v_{2}-v_{1}\right]=\lambda\left[v_{2}\right]-\lambda\left[v_{1}\right]$. Since $\lambda\left[v_{2}\right]$ $+\left|\lambda\left[v_{1}\right]\right| \leq 0$, then $\lambda\left[v_{2}\right]-\lambda\left[v_{1}\right] \leq \lambda\left[v_{2}\right]+\left|\lambda\left[v_{1}\right]\right| \leq 0$. Hence $\lambda\left[v_{2}-v_{1}\right] \leq 0$. By Lemma 2, $v_{2}-v_{1} \geq 0$ on $R_{h}$.

Also, $v_{2}+v_{1} \geq v_{2}-\left|v_{1}\right| \geq 0$ on $S_{h}$. Then, $\lambda\left[v_{2}+v_{1}\right]=\lambda\left[v_{2}\right]+\lambda\left[v_{1}\right] \leq 0$. Hence $v_{2}+v_{1} \geq 0$ on $R_{h}$. But $v_{2}+v_{1} \geq 0$ on $R_{h}$ and $v_{2}-v_{1} \geq 0$ on $R_{h}$ imply $v_{2} \geq\left|v_{1}\right|$ on $R_{h}$. This completes the proof.

LEMMA 4. If $|\lambda[v]| \leq A$ on $R_{h}$ and if $|v| \leq B$ on $S_{h}$, and $r$ is the radius of $a$ properly selected circle, as described below, which contains $G$, then $|v| \leq \frac{A}{4} r^{2}+B$. 
Proof. Let

$$
w(x, y)=\left[\frac{A r^{2}}{4}\left\{1-\frac{\left(x-x_{0}\right)^{2}+\left(y-y_{0}\right)^{2}}{r^{2}}\right\}+B\right],
$$

where $\left(x-x_{0}\right)^{2}+\left(y-y_{0}\right)^{2}=r^{2}$ is the equation of any circle containing $G$ for which $y_{0} / y \geq 1$, for all $y$ in $G$. At least one such circle exists since $G$ is simply connected, bounded, closed and $y \neq 0$, which imply that $y$ is of constant sign.

Direct calculation yields $\lambda[w]=-\frac{A}{2}\left(1+y_{0} / y\right) \leq-A$. Also, $w \geq B$ on $S_{h}$.

Now, since $|\lambda[v]| \leq A$ on $R_{h}$, by assumption, and it has been shown that $\lambda[w] \leq-A$, or equivalently, $-\lambda[w] \geq A$, it follows that $-\lambda[w] \geq-A \geq \lambda[v]$ on $R_{h}$. Since $|v| \leq B$ on $S_{h}$, and $w \geq B$ on $S_{h}, w \geq|v|$. Hence, $-|\lambda[v]| \geq \lambda[w]$ on $R_{h}$ and $w \geq|v|$ on $S_{h}$. By Lemma $3, w \geq|v|$ on $R_{h}$, or $|v| \leq w \leq \frac{A r^{2}}{4}+B$.

This completes the proof.

THEOREM. If $u(x, y)$ is of class $C^{5}$ in a closed region $G, y \neq 0$ in $G, u$ denotes the solution of the stream equation, $U$ denotes the solution of the linear system which results from application of (2.1) in the method of section $2,|\sigma|=\left|\frac{h}{y}\right|<1$, then

where

$$
|U-u| \leq \frac{r^{2}}{4}\left[\frac{h^{2} M_{3}}{3 ! \bar{y}}+\frac{h^{2} M_{4}}{3 !}+\frac{h^{4} M_{5}}{2 \cdot 4 ! \bar{y}}\right]+2 h M_{1}
$$

$$
\begin{aligned}
& M_{1}=\operatorname{Max}\left[\underset{(x, y) \in G}{\operatorname{Max}}\left|u_{1,0}\right|, \underset{(x, y) \in G}{\operatorname{Max}}\left|u_{0,1}\right|\right], \quad M_{3}=\underset{(x, y) \in G}{\operatorname{Max}}\left|u_{0,3}\right|, \\
& M_{4}=\operatorname{Max}\left[\underset{(x, y)_{e} G}{\operatorname{Max}}\left|u_{0,4}\right|, \underset{(x, y) \in G}{\operatorname{Max}}\left|u_{4,0}\right|\right], \quad M_{5}=\underset{(x, y)_{e} G}{\operatorname{Max}}\left|u_{0,5}\right|,
\end{aligned}
$$

$\bar{y}=G L B|y|$ in $G, r=$ radius of any circle of type described in Lemma 4.

Proof. Let $R=\lambda[u]-\left(u_{x x}-\frac{1}{y} u_{y}+u_{y y}\right)$. Substitution of the finite Taylor series expansions for $u_{1}, u_{2}, u_{3}, u_{4}$ in $\lambda[u]$ and use of the Mean Value Theorem yields $R=\frac{h^{2}}{4 !}\left[u_{4,0}\left(\rho_{1}, y\right)+u_{0,4}\left(x, \rho_{2}\right)+u_{4,0}\left(\rho_{3}, y\right)+u_{0,4}\left(x, \rho_{4}\right)\right]$

$$
-\frac{h^{2}}{3 ! y} u_{0,3}(x, y)+\frac{h^{4}}{2 \cdot 4 ! y}\left[u_{0,5}\left(x, \rho_{5}\right)\right]
$$

where $\rho_{2}<\rho_{5}<\rho_{4}$. Hence

$$
|R| \leq \frac{M_{4} h^{2}}{3 !}+\frac{M_{3} h^{2}}{3 ! \bar{y}}+\frac{M_{5} h^{4}}{2 \cdot 4 ! \bar{y}}
$$

Since $u_{x x}-\frac{1}{y} u_{y}+u_{y y}=0$,

$$
\left|\lambda[u]-\left(u_{x x}-\frac{1}{y} u_{y}+u_{y y}\right)\right|=|\lambda[u]|=|R| \leq \frac{h^{2} M_{3}}{3 ! \bar{y}}+\frac{h^{2} M_{4}}{3 !}+\frac{h^{4} M_{5}}{2 \cdot 4 ! \bar{y}}
$$


Also, for any point of $S_{h}, U$ was taken as the value of $g\left(x^{\prime}, y^{\prime}\right)$ at the nearest point $\left(x^{\prime}, y^{\prime}\right)$ on the boundary $S$, and $g\left(x^{\prime}, y^{\prime}\right)=u\left(x^{\prime}, y^{\prime}\right)$ on $S$. Thereby, for any point $(x, y)$ of $S_{h}$,

$$
|U(x, y)-u(x, y)|=\left|g\left(x^{\prime}, y^{\prime}\right)-u(x, y)\right|=\left|u\left(x^{\prime}, y^{\prime}\right)-u(x, y)\right|,
$$

where $\left(x^{\prime}, y^{\prime}\right)$ is a point of $S$ and $\left(x-x^{\prime}\right)^{2}+\left(y-y^{\prime}\right)^{2} \leq 2 h^{2}$. Therefore

$$
\begin{array}{r}
|U(x, y)-u(x, y)|=\left|u\left(x^{\prime}, y^{\prime}\right)-u\left(x, y^{\prime}\right)+u\left(x, y^{\prime}\right)-u(x, y)\right| \\
\leq\left|u_{x}\left(\rho_{6}, y^{\prime}\right)\left(x-x^{\prime}\right)\right|+\left|u_{y}\left(x, \rho_{7}\right)\left(y-y^{\prime}\right)\right| \\
\leq M_{1}\left[\left|y^{\prime}-y\right|+\left|x^{\prime}-x\right|\right] \leq 2 h M_{1} .
\end{array}
$$

It must also be noted that $\lambda[U]=0$, by (4.1). Hence

$$
|\lambda[u-U]|=|\lambda[u]-\lambda[U]|=|\lambda[u]| .
$$

Applying Lemma 4 to (4.8), (4.10), and (4.11), one finds that on $R_{h}$

$$
|U-u| \leq \frac{r^{2}}{4}\left\{\frac{h^{2} M_{3}}{3 ! \bar{y}}+\frac{h^{2} M_{4}}{3 !}+\frac{h^{4} M_{5}}{2 \cdot 4 ! \bar{y}}\right\}+2 h M_{1} .
$$

This completes the proof.

COROLlaRY. Under the assumptions of the theorem just proved, $U \rightarrow u$, as $h \rightarrow 0$.

Under general conditions set forth by Collatz [2], the term $2 h M_{1}$ in the error bound for $|U-u|$ may be replaced by a term of type $O\left(h^{2}\right)$ by replacing the unesthetic method for deciding $U$ on $S_{h}$ as follows. Let $\left(x_{1}, y_{1}\right)$ be a point of $S$, $\left(x_{2}, y_{2}\right)$ of $R_{h}$, and $\left(x_{0}, y_{0}\right)$ of $S_{h}$. Let $P Q$ (diagram 3) be parallel to a coordinate axis and let $\delta$ be the absolute value of the distance between $\left(x_{0}, y_{0}\right)$ and $\left(x_{1}, y_{1}\right)$. Choose $U_{0}$ by

$$
U_{0}=\frac{h g\left(x_{1}, y_{1}\right)+\delta U_{2}}{h+\delta} .
$$

5. Use of Diagonal Points. After a numerical solution has been found on a given set $G_{h}$, solutions at other points may be approximated rapidly as follows. Let $5,6,7,8$ represent four points of $G_{h}$ which are vertices of a square. (See

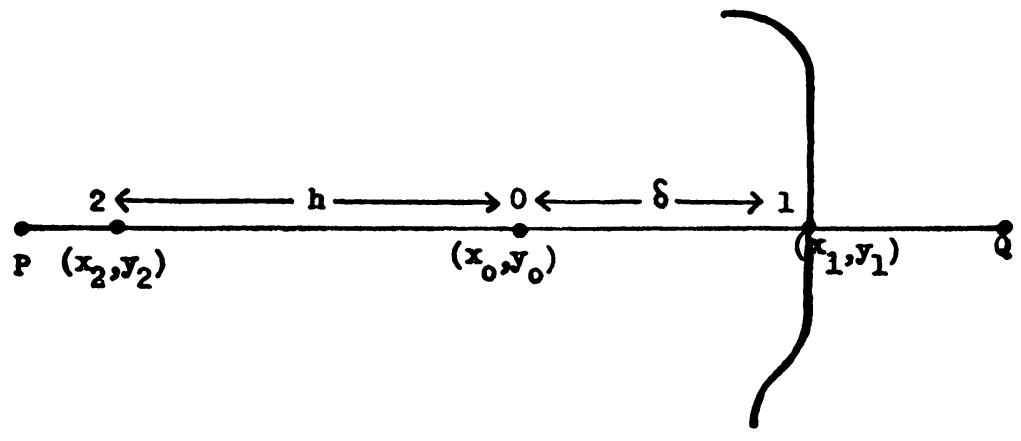

Diagrax 3 


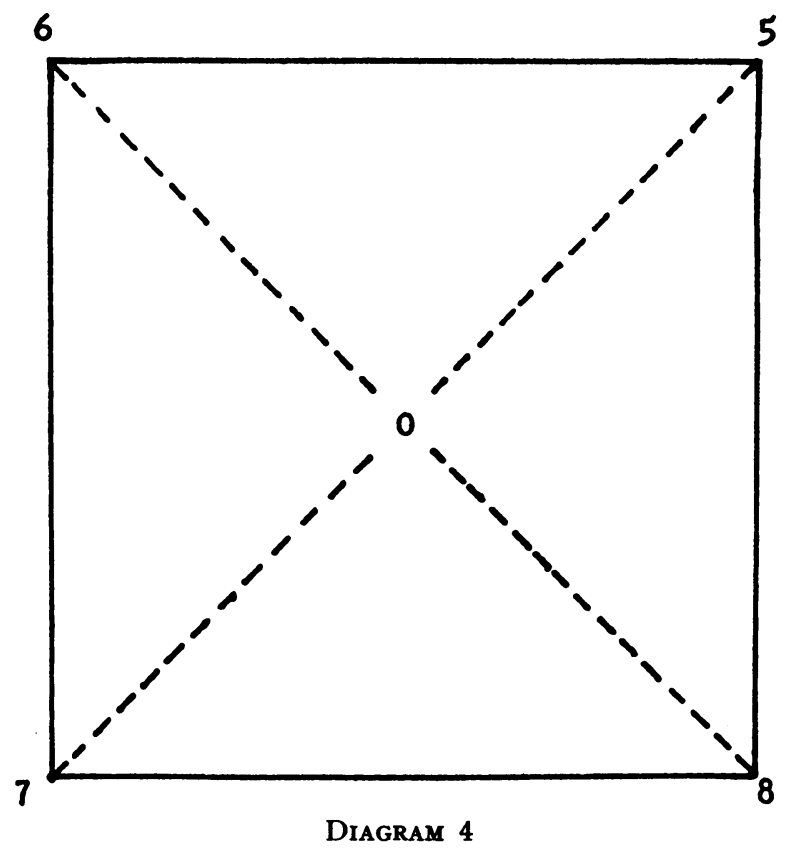

diagram 4.) Let 0 represent the diagonal point of the square. Use

$$
U_{0}=\frac{\left(2-\sigma-\sigma^{2}\right)\left(\dot{U}_{5}+U_{6}\right)+\left(2+\sigma-\sigma^{2}\right)\left(U_{7}+U_{8}\right)}{4\left(2-\sigma^{2}\right)}, \quad \sigma=\frac{h}{y_{0}} .
$$

The unexciting calculations will here be replaced by a general outline of how to establish (5.1). Let $L(u)=\alpha_{0} u_{0}+\alpha_{5} u_{5}+\alpha_{6} u_{6}+\alpha_{7} u_{7}+\alpha_{8} u_{8}$. Expand $u_{5}, u_{6}$, $u_{7}, u_{8}$ in Taylor series about $\left(x_{0}, y_{0}\right)$ and substitute these expressions into $L(u)$. Simplify by replacing $u_{i, j}$ by $i ! j ! A_{i j}$. Apply identities (4.3) and combine as in (4.4). Set the coefficients equal to $\epsilon_{i}$ 's. This system of equations is

$$
\begin{aligned}
& \alpha_{0}+\alpha_{5}+\alpha_{6}+\alpha_{7}+\alpha_{8}=\epsilon_{1}, \quad \alpha_{5}-\alpha_{6}-\alpha_{7}+\alpha_{8}=\epsilon_{2}, \quad \alpha_{5}+\alpha_{6}-\alpha_{7}-\alpha_{8}=\epsilon_{6}, \\
& \alpha_{5}\left(1+\frac{\sigma}{2}-\frac{\sigma^{2}}{2}\right)+\alpha_{6}\left(1+\frac{\sigma}{2}-\frac{\sigma^{2}}{2}\right) \\
& +\alpha_{7}\left(-1+\frac{\sigma}{2}+\frac{\sigma^{2}}{2}\right)+\alpha_{8}\left(-1+\frac{\sigma}{2}+\frac{\sigma^{2}}{2}\right)=\epsilon_{3}, \\
& \alpha_{5}\left(1+\frac{\sigma}{6}\right)-\alpha_{6}\left(1+\frac{\sigma}{6}\right)+\alpha_{7}\left(1-\frac{\sigma}{6}\right)-\alpha_{8}\left(1-\frac{\sigma}{6}\right)=\epsilon_{4},
\end{aligned}
$$

where $\epsilon_{1}=O\left(h^{4}\right), \epsilon_{2}=O\left(h^{3}\right), \epsilon_{3}=O\left(h^{3}\right), \epsilon_{4}=O\left(h^{2}\right), \epsilon_{5}=O(h)$. A general formula can be established, but by letting $\epsilon_{1}=\epsilon_{2}=\epsilon_{3}=\epsilon_{4}=0, \epsilon_{5}=h$, solving for the $\alpha_{i}$ 's and using the method of section 3, one deduces (5.1).

6. Concluding Remarks. The method and ideas developed can be used in an analogous fashion to effect the numerical solution of problems involving any one of the class of equations

$$
u_{x x}+\frac{k}{y} u_{y}+u_{y y}=0
$$


where $k$ is a fixed constant. Such equations are confronted frequently in physics (Shortley et al [8]).

Finally, two problems remain which are worthy of note. Many physical situations allow $y$ to be zero on the boundary $S$ and a solution which satisfies (6.1) in $R$ is desired. An effective technique in the sense that $U \rightarrow u$ as $h \rightarrow 0$ is needed. Also, a reasonable method for approximating the error in the numerical solution is wanting. The error bound (4.12) involves unknown quantities and the only work which has been done toward establishing error bounds which can be calculated has been for harmonic functions (Walsh and Young $[11,12]$ ).

Hughes Aircraft Company

Donald GREensPan

Culver City, California

1. D. L. BERNSTEIN, "Existence theorems in partial differential equations," Annals of Mathematics Studies, No. 23, Princeton Univ. Press, New Jersey, 1950, p. 179-192.

2. L. CollATz, "Bemerkungen zur Fehlerabschätzung für das Differenzenverfahren bei partiellen Differentialgleichungen," Zeit. angew. Math. Mech., v. 13, 1933, p. 56-57. 1937.

3. R. Courant, \& D. HILBERT, Methoden der Mathematischen Physik, Julius Springer, Berlin,

4. H. GeIRINGER, "On the solution of systems of linear equations by certain iteration methods," Reissner Anniv. Volume, Ann Arbor, Michigan, 1949, p. 365-393.

5. S. GERSCHGORIN, "Fehlerabschätzung für das Differenzenverfahren zur Lösung partieller Differentialgleichungen," Zeit. angew. Math. Mech., v. 10, 1930, p. 373-382.

6. D. GREENSPAN, "Methods of matrix inversion," Amer. Math. Mon., v. 62, 1955, p. 303-318.

7. W. E. MILNE, Numerical Solution of Differential Equations, John Wiley \& Sons, Inc., New York, 1953.

8. G. Shortley, R. Weller, P. Darby, \& E. H. Gamble, "Numerical solution of axisymmetrical problems with applications to electrostatics and torsion," Jn. Appl. Phys., v. 18, 1947, p. 116-129.

9. NBS Applied Mathematics Series, No. 29, Simultaneous Linear Equations and the Determination of Eigenvalues, U. S. Gov. Printing Office, Washington, D. C., 1953.

10. G. TEMPLE, "The general theory of relaxation methods applied to linear systems," Roy. Soc. London, Proc., v. 169, Ser. A, 1939, p. 476-500.

11. J. L. WALSH \& D. Young, "On the accuracy of the numerical solution of the Dirichlet problem by finite differences," NBS Jn. of Research, v. 51, 1953, p. 343-363.

12. J. L. WALSH \& D. Young, "On the degree of convergence of solutions of difference equations to the solution of the Dirichlet problem," Jn. Math. and Physics, v. 33, 1954, p. 80-93.

13. D. M. Young, "Numerical methods for solving partial differential equations," Mimeographed lecture notes, course at Ballistics Institute, Ball. Res. Lab., Aberdeen Proving Grounds, Maryland, 1951-1952.

14. D. M. YounG, "On the solution of linear systems by iteration," Prelim. Report No. 9, Army Office of Ordnance Research, Project No. TB-2-0001 (407) with the Univ. of Maryland, 1953.

15. D. M. YounG, "Iterative methods for solving partial difference equations of elliptic type,"

Amer. Math. Soc., Trans., v. 76, 1954, p. 92-111.

\section{Tables of Values of 16 Integrals of Algebraic- Hyperbolic Type}

This paper gives tables of values of the following 16 integrals of algebraichyperbolic type.

$$
\begin{array}{cl}
I_{k}=\frac{2^{k}}{k !} \int_{0}^{\infty} \frac{x^{k} d x}{\sinh 2 x \pm 2 x}, & (k \geqslant 1) \\
I_{k}{ }^{*} & (k \geqslant 3) \\
I I_{k}=\frac{2^{k}}{k !} \int_{0}^{\infty} \frac{x^{k} e^{-2 x} d x}{\sinh 2 x \pm 2 x}, \quad(k \geqslant 1) \\
I I_{k}{ }^{*}, \quad(k \geqslant 3)
\end{array}
$$

